\title{
Analog of microwave-induced resistance oscillations induced in GaAs heterostructures by terahertz radiation
}

\author{
T. Herrmann, ${ }^{1}$ I. A. Dmitriev, ${ }^{2,3}$ D. A. Kozlov, ${ }^{4,5}$ M. Schneider, ${ }^{1}$ B. Jentzsch, ${ }^{1}$ Z. D. Kvon,,${ }^{4,5}$ P. Olbrich, ${ }^{1}$ V. V. Bel'kov, ${ }^{3}$ \\ A. Bayer, ${ }^{1}$ D. Schuh, ${ }^{1}$ D. Bougeard, ${ }^{1}$ T. Kuczmik, ${ }^{1}$ M. Oltscher, ${ }^{1}$ D. Weiss, ${ }^{1}$ and S. D. Ganichev ${ }^{1}$ \\ ${ }^{1}$ Terahertz Center, University of Regensburg, 93040 Regensburg, Germany \\ ${ }^{2}$ Max Planck Institute, 70569 Stuttgart, Germany \\ ${ }^{3}$ Ioffe Institute, 194021 St. Petersburg, Russia \\ ${ }^{4}$ Rzhanov Institute of Semiconductor Physics, 630090 Novosibirsk, Russia \\ ${ }^{5}$ Novosibirsk State University, 630090 Novosibirsk, Russia
}

(Received 5 March 2016; published 1 August 2016)

\begin{abstract}
We report on the study of terahertz radiation-induced MIRO-like oscillations of magnetoresistivity in GaAs heterostructures. Our experiments provide an answer on two most intriguing questions-effect of radiation helicity and the role of the edges-yielding crucial information for an understanding of the MIRO (microwave-induced resistance oscillations) origin. Moreover, we demonstrate that the range of materials exhibiting radiation-induced magneto-oscillations can be largely extended by using high-frequency radiation.
\end{abstract}

DOI: 10.1103/PhysRevB.94.081301

One of the most interesting phenomena recently observed in two-dimensional electron systems (2DES) is microwave (MW) -induced resistance oscillations (MIRO) and associated zero resistance states (ZRS) [1-7], reviewed, e.g., in [8]. Like Shubnikov-de Haas oscillations $(\mathrm{SdH}), \mathrm{MIRO}$ are periodic on a $1 / B$ scale, but occur at lower magnetic fields and show much weaker temperature dependence. Phenomenologically, they are very similar to Weiss oscillations [9], which reflect the commensurability between the cyclotron orbit radius and the period of a periodic potential. MIRO by contrast, reflect the commensurability between the MW photon energy $2 \pi \hbar f$ and the cyclotron energy $\hbar \omega_{c}$. In extremely clean samples the minima of the MIRO develop into ZRS [3-5], which are explained [10] in terms of an instability of the system and formation of current domains, occurring when the conductivity becomes negative under MW irradiation (see also $[8,11,12]$ ).

In spite of numerous experiments and significant advances in their theoretical understanding, there is still no commonly accepted microscopic description of the effect [13,14] and the ongoing MIRO investigations remain challenging [15-21]. Consequently, new materials have been studied [22-25] and new theoretical models have been put forward [26-30]. To the most pressing issues which need to be clarified experimentally and which might help to differentiate between the different models belong the MIRO polarization dependence $[7,8,31]$ and the "bulk" or "edge" nature of the effect.

So far the majority of experimental work has been done in the MW regime (1-350 GHz) and there are only a few reports on MIRO excited at terahertz (THz) frequencies [32-34]. Here we report on the observation of pronounced MIRO-like oscillations induced by $\mathrm{THz}$ radiation. We exploit the specific advantages of $\mathrm{THz}$ laser radiation not present in the $\mathrm{MW}$ regime, i.e., the possibility to focus it onto a spot smaller than the sample's size and easy control of the radiation's polarization. The most important features clearly detected on a large variety of samples are (i) a very weak dependence of the oscillations' amplitude on the photon helicity and (ii) the bulk nature of the effect. Furthermore, our study shows that the MIRO oscillations can be excited at $\mathrm{THz}$ frequencies even in the samples with low mobility, whereas in the MW range ultrahigh mobility samples are crucially needed for this type of experiments.

We study the radiation-induced oscillations in AlGaAs/GaAs quantum wells of 10 or $12.5 \mathrm{~nm}$ thickness. While THz MIRO oscillations have been observed in Hall bar samples [33], in this work we used Corbino disk samples, which measure directly $\sigma_{x x}$. The inner, $r_{i}$, and the outer, $r_{o}$, radius of the gold-germanium contacts and samples' transport parameters are given in Table I (see also the Supplemental Material [35]). The effect of $\mathrm{THz}$ radiation on conductivity $\sigma_{x x}$ was studied in the temperature range from $T=2.5 \mathrm{~K}$ to about $20 \mathrm{~K}$. The MIRO effect is most pronounced after the cooled samples have been exposed to room light, but the effect is also present for samples kept in the dark. All data presented in figures and tables were obtained after sample illumination.

The $\mathrm{THz}$ experiments were performed using continuous wave (cw) $\mathrm{CH}_{2} \mathrm{O}_{2}$ and $\mathrm{CH}_{2} \mathrm{~F}_{2}$ lasers [36-38] operating at frequencies $f=0.69$ and $1.63 \mathrm{THz}$, respectively. The radiation at normal incidence is focused onto a spot size of about $3.4 \mathrm{~mm}$ diameter. The beam has an almost Gaussian profile, measured by a pyroelectric camera $[39,40]$. Right- and left-handed circularly polarized radiation is obtained by a $\lambda / 4$ quartz plate [41-43]. The inset in Fig. 1(b) sketches the setup. Applying a bias voltage, $U_{\mathrm{dc}}=50 \mathrm{mV}$, and measuring the photovoltage $U$ on the load resistor $R_{L}=50 \Omega$ we obtained the photoconductivity $\Delta \sigma_{x x} \propto U$.

Irradiating the samples with $\mathrm{THz}$ radiation and sweeping the magnetic field $B$ results in conductivity oscillations $\Delta \sigma_{x x}$. Figure 1 shows the photoconductivity (PC) for sample A and $f=0.69 \mathrm{THz}$. The oscillations reflect two $1 / B$ periodicities: one at low $B$ corresponding to the MIRO oscillations and the other, at higher fields $|B| \sim 1 \mathrm{~T}$, corresponding to $\mathrm{SdH}$ oscillations. While the former oscillations are detected up to temperatures of about $20 \mathrm{~K}$, the latter are observed at substantially lower temperatures only. The oscillations are observed for structures with mobilities between $\mu=1.8 \times 10^{6}$ and $1.5 \times 10^{5} \mathrm{~cm}^{2} / \mathrm{V}$ s and with electron densities from $n_{e}=4$ to $24 \times 10^{11} \mathrm{~cm}^{-2}$. Figure 2 shows $\Delta \sigma_{x x}$ normalized to the 


\section{T. HERRMANN et al.}

TABLE I. Samples and their transport data at $T=2 \mathrm{~K}$ including the electron density $n_{e}$ and mobility $\mu$, as well as the momentum, $\tau_{p}$, and quantum, $\tau_{q}$, relaxation times.

\begin{tabular}{llccccr}
\hline \hline Sample & $\begin{array}{c}r_{i} \\
(\mathrm{~mm})\end{array}$ & $\begin{array}{c}r_{o} \\
(\mathrm{~mm})\end{array}$ & $\begin{array}{c}\mu \\
10^{3}\left(\mathrm{~cm}^{2} / \mathrm{Vs}\right)\end{array}$ & $\begin{array}{c}n_{e} \\
10^{11}\left(\mathrm{~cm}^{-2}\right)\end{array}$ & $\begin{array}{c}\tau_{p} \\
(\mathrm{ps})\end{array}$ & $\begin{array}{c}\tau_{q} \\
(\mathrm{ps})\end{array}$ \\
\hline A & 0.25 & 1.5 & 820 & 12.0 & 33 & 4.2 \\
$\mathrm{~B}$ & 0.3 & 1.0 & 1800 & 9.3 & 71 & 12.0 \\
$\mathrm{C}$ & 0.3 & 1.0 & 150 & 18.0 & 6 & 1.3 \\
$\mathrm{D}$ & 0.25 & 1.5 & 980 & 24.0 & 39 & 1.9 \\
$\mathrm{E}$ & 0.25 & 1.5 & 280 & 3.7 & 11 & 4.1 \\
\hline \hline
\end{tabular}

dark conductivity, $\Delta \sigma_{x x} / \sigma_{x x}$, for different samples. The data clearly show that the oscillations are periodic in $\epsilon=\omega / \omega_{c}$ with $\omega=2 \pi f$ and exhibit extrema at $\epsilon=\epsilon_{N} \pm 1 / 4$, where $\epsilon_{N} \simeq N=1,2,3 \ldots$ denote the position of the nodes $[44,45]$ and $+(-)$ corresponds to minima (maxima). Here, the position of the cyclotron resonance $(\mathrm{CR})$ at $\omega=\omega_{c}$ has been obtained from the transmission experiments displayed in the inset of Fig. 1(a). The overall behavior of the observed $1 / B$ oscillations including the exponential damping at low $B$ and the $1 / 4$ shift of the minima and maxima from the nodes corresponds to the well known MIRO effects as is further discussed below. Strikingly, Figs. 1 and 2 reveal that the oscillation amplitudes depend only weakly on the radiation helicity and some samples (B and D)
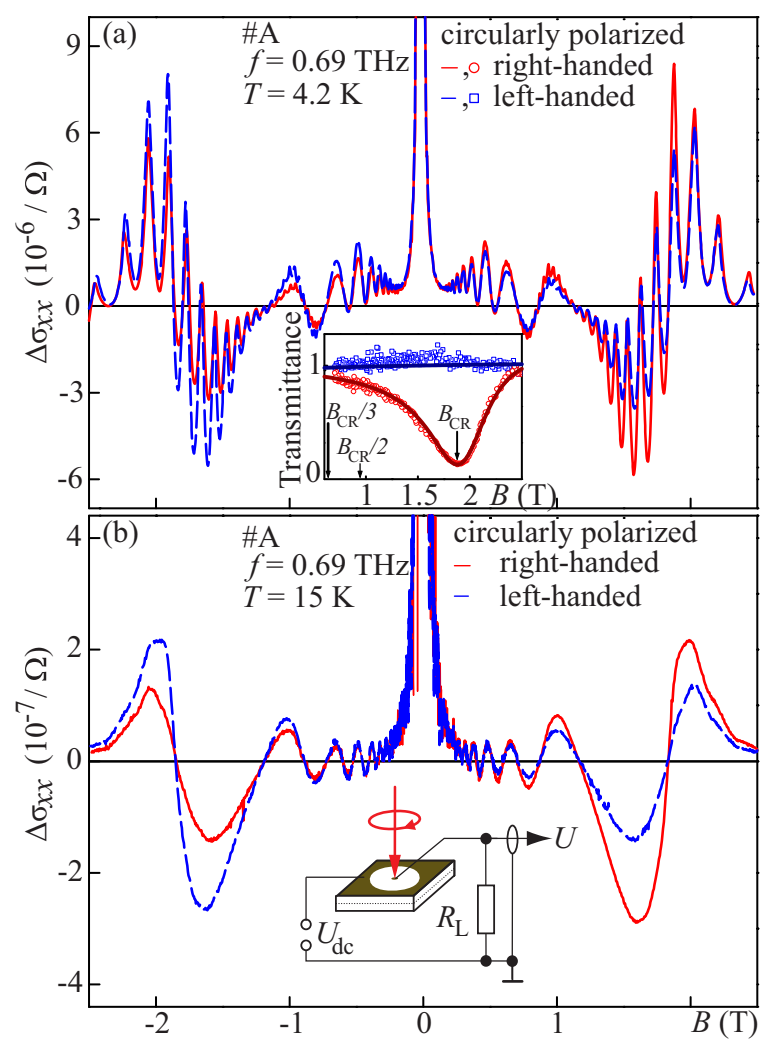

FIG. 1. Magnetic field dependence of $\Delta \sigma_{x x}$ induced by modulated $\mathrm{cw}$ radiation with power $P \approx 10 \mathrm{~mW}$. The inset in panel (a) shows the transmission data. Lines show the transmission calculated after [47] by taking multiple reflections within the substrate and the superradiant decay into account [47-50].
PHYSICAL REVIEW B 94, 081301(R) (2016)

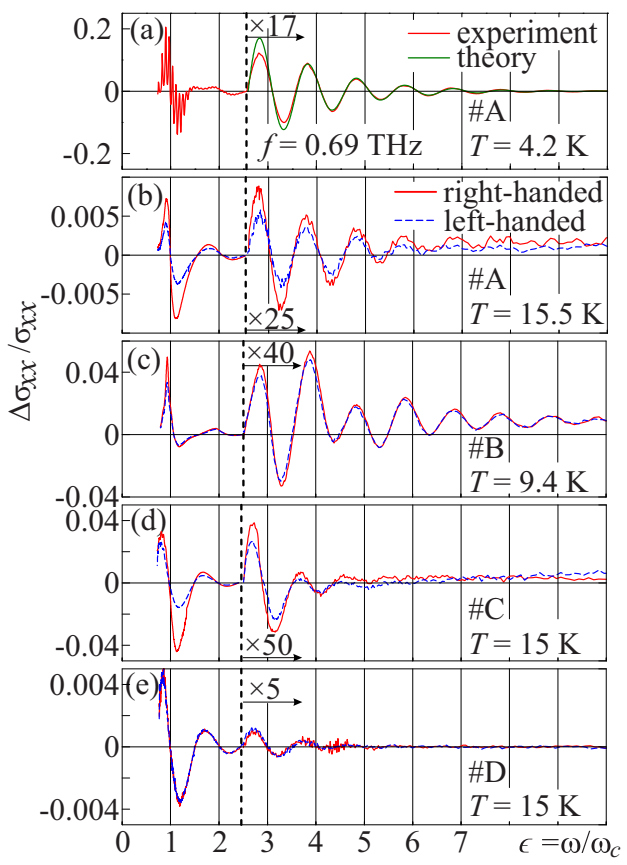

FIG. 2. $\Delta \sigma_{x x} / \sigma_{x x}$ as a function of $\omega / \omega_{c}$. The green line in (a) is a fit of the low- $B$ tail of the oscillations using Eq. (1) with a $B$-independent $A_{\epsilon}=A_{\infty}$ [44]. Such fit yields $A_{\infty}^{\text {expt. }}=0.056$ in agreement with theory [52] yielding $A_{\infty}^{\text {theor }} \sim 0.04$. The monotonic part of $\Delta \sigma_{x x} / \sigma_{x x}$ in panel (a) is subtracted. Data for $\epsilon>2.5$ are magnified by the factors shown in the panels.

show nearly no helicity dependence. By contrast, transmission, displayed in the inset in Fig. 1(a), strongly depends on helicity. CR for positive $B$ occurs only for right-handed circular polarization and vice versa. Indeed, in agreement with the theory of CR [46], the transmitted power drops nearly to zero for the cyclotron resonance active configuration (CRA), whereas the signal for the opposite helicity, i.e., for the inactive configuration (CRI), is almost $B$ independent. Active and inactive helicity interchange for negative magnetic fields (not shown). Note that the detected polarization behavior and the shape of transmittance minimum are well fitted by taking multiple reflections in the substrate and superradiant decay into account [47-50] (see solid lines in the inset of Fig. 1(a) and the Supplemental Material [35]). To demonstrate that the weak polarization dependence of the MIRO oscillations is not caused by saturation effects we measured $\Delta \sigma_{x x}$ at different power levels. These data show [35] that the signal scales linearly with power and reducing the power by ten times does not change the ratio between CRA and CRI signals. The overall same results are obtained for $f=1.63 \mathrm{THz}$ [35]. The only differences are the signal magnitude and rescaled $B$-field positions of $\mathrm{CR}$ and oscillations.

The results shown in Figs. 1 and 2 are obtained for Corbino disks with an outer diameter $3 \mathrm{~mm}$, thus being of the order of the laser spot size. To exclude possible effects stemming from irradiation of the contacts' edges we carried out measurements on disks with $8.5 \mathrm{~mm}$ diameter, i.e., significantly larger than the spot size. These samples are indicated by a subscript $\mathrm{L}$ in the sample index. Figures 3(a) and 3(b) show that the oscillations can be efficiently excited without $\mathrm{THz}$ irradiation of the 


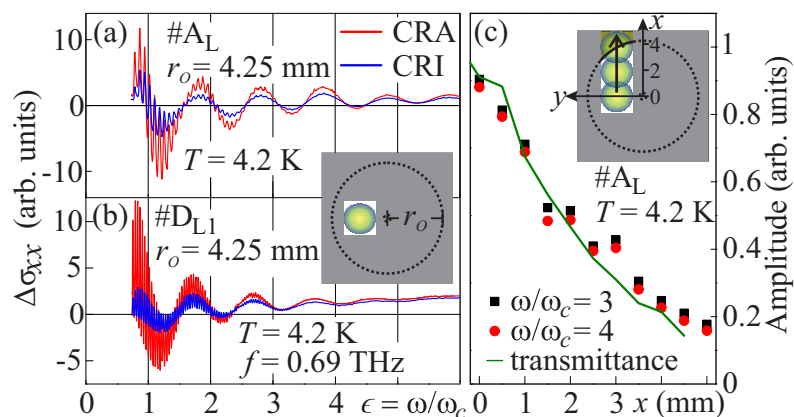

FIG. 3. $\Delta \sigma_{x x}$ as a function of $\omega / \omega_{c}$ for large size samples $A_{\mathrm{L}}$ (a) and $\mathrm{D}_{\mathrm{L} 1}$ (b) with a metal mask (gray square) preventing irradiation of contacts. (c) Oscillation amplitude as a function of the laser spot position for sample covered by a metal mask with a slit (see inset). The beam is scanned along the slit from bulk regions of the Corbino disk onto the outer contact area. The solid line shows the transmission signal measured simultaneously with the oscillations.

contacts. We measured the photoconductivity under irradiation of the sample through a hole in a metal mask covering the contacts. The mask was mounted at a distance $\approx 1 \mathrm{~mm}$ from the sample surface [see inset in Fig. 3(a)]. Furthermore, we removed a part of the metal screen and scanned the beam across the edge [see inset in Fig. 3(c)]. Figure 3(c) shows that the signal decreases as the laser beam approaches the edge, demonstrating that the oscillation amplitude just follows the decrease in absorbed radiation power. Hence, the data directly show that oscillations are dominated by bulk effects in 2DES and, at least in the $\mathrm{THz}$ range, do not come from the edge phenomena such as ponderomotive forces excited by contacts/edge illumination [29] or radiationinduced modification of the edge transport [30]. Importantly, the polarization dependence of the oscillations remains weak for any position of the laser spot including both geometries, where the contacts are avoided and where the beam is focused on the edge. This is illustrated in Fig. 4, where the ratios of the oscillation amplitude for CRA and CRI configurations are

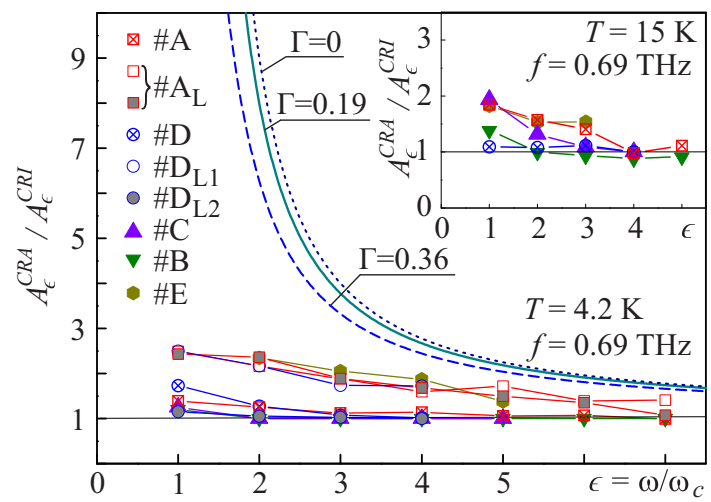

FIG. 4. $A_{\epsilon}^{\mathrm{CRA}} / A_{\epsilon}^{\mathrm{CRI}}$ vs $\epsilon$ for all samples. For sample $\mathrm{A}_{\mathrm{L}}$ it is shown for the laser focused in between the contacts (open squares) and onto the edge of the outer Corbino contact (solid squares). $\mathrm{D}_{\mathrm{L} 1}$ and $\mathrm{D}_{\mathrm{L} 2}$ are large samples made from the same wafer. Lines are calculated using Eq. (3) for $\Gamma=0.19$ and 0.36 corresponding to samples A and $\mathrm{D}$, respectively. shown as a function of $\omega / \omega_{c}$. In all cases and especially for low oscillation indices the experimental CRA/CRI ratio is always much smaller than the calculated one, shown for different radiative decay $\Gamma$ (discussed below) in Fig. 4. The finding that the $\mathrm{THz}$-induced oscillations depend only weakly on helicity is in line with the results of Smet et al., who examined MIROs at MW frequencies [7].

Now we discuss the results within the inelastic $[6,51,52]$ and the displacement [53-57] mechanisms, which ascribe the magneto-oscillations of the PC to radiation-assisted scattering between disorder-broadened Landau levels. This theory is a nonequilibrium extension of linear transport theory in high Landau levels [58], and accounts for both real-space displacements of electron orbits in individual photon-assisted scattering events (displacement contribution) and associated nonequilibrium occupation of electronic states within the disorder-broadened Landau levels (inelastic contribution). Within this theory the conductivity oscillations are given by [59]

$$
\Delta \sigma_{x x} / \sigma_{x x}=-\epsilon A_{\epsilon} \sin (2 \pi \epsilon) \exp (-\alpha \epsilon),
$$

where the exponential damping with $\alpha=2 \pi / \omega \tau_{q}$ corresponds to the dirty limit $\omega_{c} \tau_{q} \ll 1$ of strongly overlapping Landau levels. Importantly, the factor $A_{\epsilon}$ becomes $B$ independent for $\epsilon=\omega / \omega_{c} \gg 1$. Fitting the low- $B$ data in Fig. 2(a) using Eq. (1) with constant $A_{\infty}=\left.A_{\epsilon}\right|_{\epsilon \rightarrow \infty}$ reveals that the experimental oscillation amplitude, $A_{\infty}^{\text {expt. }}=0.056$, agrees well with the theoretical predictions for the inelastic mechanism [52], $A_{\infty}^{\text {theor }} \sim 0.04$ (see [44] and the Supplemental Material [35]). This shows, in particular, that $\Delta \sigma_{x x}$ follows the $n_{e}^{3} / \omega^{4}$ dependence predicted for a dominant inelastic mechanism. In accordance with Eq. (1), the number of resolved oscillations for all samples in Fig. 2 is roughly equal to $\omega \tau_{q} / 2$. Here $\tau_{q}$ are taken from the analysis of SdH oscillations (see Table I). This highlights the important role of $\tau_{q}$ in the appearance of oscillations and explains why at $\mathrm{THz}$ frequencies they can be observed in samples with relatively low mobility.

While the inelastic mechanism of MIRO describes the experimental findings for large $\omega / \omega_{c}$ quite well, it cannot explain the weak polarization dependence measured for $\omega / \omega_{c} \leqslant 5$. In theory, the polarization dependence of $\Delta \sigma_{x x}$ follows the absorbance $K=\sigma_{x x}^{( \pm)}(\omega) E_{ \pm}^{2} / 2$, where $\sigma_{x x}^{( \pm)}(\omega)$ is the dissipative part of the linear dynamic conductivity, $E_{ \pm}$ is the amplitude of the screened radiation electric field, and $+(-)$ labels the CRI (CRA) configuration [60,61]. Although the magneto-oscillations in $\Delta \sigma_{x x}$ are of quantum origin, their polarization dependence stems from Drude theory $[62,63]$ and reflects the classical dynamics of $2 \mathrm{DES}$ in crossed magnetic field $B$ and electric field of the THz wave, yielding [46]

$$
A_{\epsilon} \propto K \propto \frac{E_{ \pm}^{2}}{\left(1 \pm\left|\omega_{c}\right| / \omega\right)^{2}+\gamma^{2}}, \quad \gamma=1 / \omega \tau_{p} \ll 1 .
$$

In order to relate $E_{ \pm}$, acting on the electrons in 2DES, to the electric field $E_{ \pm}^{(0)}$ of the incoming wave, one needs to take into account both the dynamical screening by the 2DES and multiple reflections within the dielectric substrate (FabryPerot interference). The former is dominated by the Hall part $\sigma_{x y}^{( \pm)}(\omega)$ of the dynamic conductivity and is described by the superradiant decay rate $\Gamma=e^{2} n_{e} / 2 \omega \epsilon_{0} m c \gg \gamma$ [47-50]. The 
effect of the dynamical screening is most simply expressed in the case of constructive Fabry-Perot interference [64]: the absorbance still has the form of Eq. (2) where $E_{ \pm}$get replaced by $E_{ \pm}^{(0)}$ and $\gamma$ by $(\Gamma+\gamma)$. Then, since $\Gamma \gg \gamma$, the ratio of the CRA and CRI absorption coefficient and oscillation amplitude is given by

$$
\frac{K^{\mathrm{CRA}}}{K^{\mathrm{CRI}}}=\frac{A_{\epsilon}^{\mathrm{CRA}}}{A_{\epsilon}^{\mathrm{CRI}}} \simeq \frac{\left(1+\left|\omega_{c}\right| / \omega\right)^{2}+\Gamma^{2}}{\left(1-\left|\omega_{c}\right| / \omega\right)^{2}+\Gamma^{2}} .
$$

Note that the value of $\Gamma$ is fully determined by $n_{e}$ and $m$ known precisely from independent measurements. Taking multiple reflections into account (see Supplemental Material [35]), the transmission data for CRA and CRI polarization can be perfectly fitted using the above expression for $\Gamma$ [see inset in Fig. 1(a)]. In Fig. 4 we present the ratio (3) calculated for different samples. The smallest $A_{\epsilon}^{\mathrm{CRA}} / A_{\epsilon}^{\mathrm{CRI}}$ value is obtained for the largest $\Gamma$, in our case $\Gamma=0.36$ for sample $\mathrm{D}$ with the largest carrier density $n_{e}=2.4 \times 10^{12} \mathrm{~cm}^{-2}$ (see dashed line in Fig. 4). Clearly, for low harmonics $\omega / \omega_{c} \leqslant 5$, the theoretical predictions for $A_{\epsilon}^{\mathrm{CRA}} / A_{\epsilon}^{\mathrm{CRI}}$ strongly deviate from the experimental data in Fig. 4. Unreasonably high values of $\Gamma \geqslant 1.5$ would be needed to match experiment. For higher harmonics, however, i.e., for $\omega / \omega_{c} \gtrsim 5, A_{\epsilon}^{\mathrm{CRA}} / A_{\epsilon}^{\mathrm{CRI}} \rightarrow 1$ both in theory and experiment. As detailed in the Supplemental Material [35], the data in this low- $B$ region are in good quantitative agreement with theory.

To summarize, our experiments provide clear evidence that MIRO oscillations in the THz regime are excited in the "bulk" of $2 \mathrm{DES}$ and not at contacts/sample boundaries. In the $\mathrm{THz}$ range they are also observed at much lower mobilities of the 2DES as compared to the MW regime suggesting that they appear at $\omega \gtrsim \tau_{q}^{-1}$. Although at high harmonics of the cyclotron resonance the observed MIRO oscillations are well described by the inelastic mechanism [52], their polarization dependence at low harmonics is at odds with any existing theoretical description of MIRO.

\section{ACKNOWLEDGMENTS}

Financial support via the German Science Foundation DFG, the Volkswagen Stiftung Program, RFBR (N 14-02-01246), and RAS is gratefully acknowledged.
[1] M. A. Zudov, R. R. Du, J. A. Simmons, and J. L. Reno, Phys. Rev. B 64, 201311(R) (2001).

[2] P. D. Ye, L. W. Engel, D. C. Tsui, J. A. Simmons, J. R. Wendt, G. A. Vawter, and J. L. Reno, Appl. Phys. Lett. 79, 2193 (2001).

[3] R. G. Mani, J. H. Smet, K. von Klitzing, V. Narayanamurti, W. B. Johnson, and V. Umansky, Nature (London) 420, 646 (2002).

[4] M. A. Zudov, R. R. Du, L. N. Pfeiffer, and K. W. West, Phys. Rev. Lett. 90, 046807 (2003).

[5] C. L. Yang, M. A. Zudov, T. A. Knuuttila, R. R. Du, L. N. Pfeiffer, and K. W. West, Phys. Rev. Lett. 91, 096803 (2003).

[6] S. I. Dorozhkin, JETP Lett. 77, 577 (2003).

[7] J. H. Smet, B. Gorshunov, C. Jiang, L. Pfeiffer, K. West, V. Umansky, M. Dressel, R. Meisels, F. Kuchar, and K. von Klitzing, Phys. Rev. Lett. 95, 116804 (2005).

[8] I. A. Dmitriev, A. D. Mirlin, D. G. Polyakov, and M. A. Zudov, Rev. Mod. Phys. 84, 1709 (2012).

[9] D. Weiss, K. von Klitzing, K. Ploog, and G. Weimann, Europhys. Lett. 8, 179 (1989).

[10] A. V. Andreev, I. L. Aleiner, and A. J. Millis, Phys. Rev. Lett. 91, 056803 (2003).

[11] I. A. Dmitriev, M. Khodas, A. D. Mirlin, and D. G. Polyakov, Phys. Rev. Lett. 111, 206801 (2013).

[12] S. I. Dorozhkin, V. Umansky, L. N. Pfeiffer, K. W. West, K. Baldwin, K. von Klitzing, and J. H. Smet, Phys. Rev. Lett. 114, 176808 (2015).

[13] M. A. Zudov, Phys. Rev. B 92, 047301 (2015).

[14] S. A. Mikhailov, Phys. Rev. B 92, 047302 (2015).

[15] Q. Shi, M. Khodas, A. Levchenko, and M. A. Zudov, Phys. Rev. B 88, 245409 (2013).

[16] A. D. Levin, Z. S. Momtaz, G. M. Gusev, and A. K. Bakarov, Phys. Rev. B 89, 161304(R) (2014).

[17] S. Chakraborty, A. T. Hatke, L. W. Engel, J. D. Watson, and M. J. Manfra, Phys. Rev. B 90, 195437 (2014).
[18] Q. Shi, P. D. Martin, A. T. Hatke, M. A. Zudov, J. D. Watson, G. C. Gardner, M. J. Manfra, L. N. Pfeiffer, and K. W. West, Phys. Rev. B 92, 081405(R) (2015).

[19] A. D. Levin, Z. S. Momtaz, G. M. Gusev, O. E. Raichev, and A. K. Bakarov, Phys. Rev. Lett. 115, 206801 (2015).

[20] A. A. Bykov, I. S. Strygin, A. V. Goran, A. K. Kalagin, E. E. Rodyakina, and A. V. Latyshev, Appl. Phys. Lett. 108, 012103 (2016).

[21] S. A. Studenikin, A. S. Sachrajda, J. A. Gupta, Z. R. Wasilewski, O. M. Fedorych, M. Byszewski, D. K. Maude, M. Potemski, M. Hilke, K. W. West, and L. N. Pfeiffer, Phys. Rev. B 76, 165321 (2007).

[22] M. A. Zudov, O. A. Mironov, Q. A. Ebner, P. D. Martin, Q. Shi, and D. R. Leadley, Phys. Rev. B 89, 125401 (2014).

[23] Q. Shi, Q. A. Ebner, and M. A. Zudov, Phys. Rev. B 90, 161301 (2014).

[24] R. Yamashiro, L. V. Abdurakhimov, A. O. Badrutdinov, Yu. P. Monarkha, and D. Konstantinov, Phys. Rev. Lett. 115, 256802 (2015).

[25] D. F. Karcher, A. V. Shchepetilnikov, Yu. A. Nefyodov, J. Falson, I. A. Dmitriev, Y. Kozuka, D. Maryenko, A. Tsukazaki, S. I. Dorozhkin, I. V. Kukushkin, M. Kawasaki, and J. H. Smet, Phys. Rev. B 93, 041410(R) (2016).

[26] Y. M. Beltukov and M. I. Dyakonov, Phys. Rev. Lett. 116, 176801 (2016).

[27] V. A. Volkov and A. A. Zabolotnykh, Phys. Rev. B 89, 121410(R) (2014).

[28] O. V. Zhirov, A. D. Chepelianskii, and D. L. Shepelyansky, Phys. Rev. B 88, 035410 (2013).

[29] S. A. Mikhailov, Phys. Rev. B 83, 155303 (2011).

[30] A. D. Chepelianskii and D. L. Shepelyansky, Phys. Rev. B 80, 241308(R) (2009).

[31] T. Ye, H.-C. Liu, Z. Wang, W. Wegscheider, and R. G. Mani, Sci. Rep. 5, 14880 (2015). 
[32] A. Wirthmann, B. D. McCombe, D. Heitmann, S. Holland, K. J. Friedland, and Can-Ming Hu, Phys. Rev. B 76, 195315 (2007).

[33] Z. D. Kvon, D. A. Kozlov, S. N. Danilov, C. Zoth, P. Vierling, S. Stachel, V. V. Bel'kov, A. K. Bakarov, D. V. Dmitriev, A. I. Toropov, and S. D. Ganichev, JETP Lett. 97, 41 (2013).

[34] R. G. Mani, A. N. Ramanayaka, Tianyu Ye, M. S. Heimbeck, H. O. Everitt, and W. Wegscheider, Phys. Rev. B 87, 245308 (2013).

[35] See Supplemental Material at http://link.aps.org/supplemental/ 10.1103/PhysRevB.94.081301 for the details of the transmission data analysis, comparison of experimental results for MIROlike oscillations with the theory of inelastic mechanism, linear magnetotransport data, and additional results on $\mathrm{THz}$ radiationinduced resistance oscillations.

[36] S. D. Ganichev, S. A. Tarasenko, V. V. Bel'kov, P. Olbrich, W. Eder, D. R. Yakovlev, V. Kolkovsky, W. Zaleszczyk, G. Karczewski, T. Wojtowicz, and D. Weiss, Phys. Rev. Lett. 102, 156602 (2009).

[37] S. D. Ganichev, I. N. Yassievich, W. Prettl, J. Diener, B. K. Meyer, and K. W. Benz, Phys. Rev. Lett. 75, 1590 (1995).

[38] P. Olbrich, C. Zoth, P. Lutz, C. Drexler, V. V. Bel'kov, Ya. V. Terent'ev, S. A. Tarasenko, A. N. Semenov, S. V. Ivanov, D. R. Yakovlev, T. Wojtowicz, U. Wurstbauer, D. Schuh, and S. D. Ganichev, Phys. Rev. B 86, 085310 (2012).

[39] S. D. Ganichev, Physica B 273-274, 737 (1999).

[40] E. Ziemann, S. D. Ganichev, I. N. Yassievich, V. I. Perel, and W. Prettl, J. Appl. Phys. 87, 3843 (2000).

[41] S. D. Ganichev and W. Prettl, Intense Terahertz Excitation of Semiconductors (Oxford University Press, Oxford, 2006).

[42] V. V. Bel'kov and S. D. Ganichev, Semicond. Sci. Technol. 23, 114003 (2008).

[43] P. Olbrich, E. L. Ivchenko, R. Ravash, T. Feil, S. D. Danilov, J. Allerdings, D. Weiss, D. Schuh, W. Wegscheider, and S. D. Ganichev, Phys. Rev. Lett. 103, 090603 (2009).

[44] In our experiments, the positions of the nodes deviate a bit from the integer values of $\epsilon$ being established in both theory and previous MW measurements [45, 8]. Accordingly, the data is fitted using Eq. (1) with $\sin (2 \pi \epsilon)$ replaced by $\sin \left(2 \pi\left[\epsilon+\epsilon_{0}\right]\right)$. The small shifts $\epsilon_{0}$ varying from sample to sample both in sign and magnitude (see Supplemental Material [35]) are likely to be of instrumental origin and should be disregarded. In Fig. 2(a), $\epsilon_{0}=-0.09$.

[45] R. G. Mani, J. H. Smet, K. von Klitzing, V. Narayanamurti, W. B. Johnson, and V. Umansky, Phys. Rev. Lett. 92, 146801 (2004).
[46] K. Seeger, Semiconductor Physics: An Introduction (Springer, New York, 2004).

[47] G. Abstreiter, J. P. Kotthaus, J. F. Koch, and G. Dorda, Phys. Rev. B 14, 2480 (1976).

[48] K. W. Chiu, T. K. Lee, and J. J. Quinn, Surf. Sci. 58, 182 (1976).

[49] S. A. Mikhailov, Phys. Rev. B 70, 165311 (2004).

[50] Qi Zhang, T. Arikawa, E. Kato, J. L. Reno, Wei Pan, J. D. Watson, M. J. Manfra, M. A. Zudov, M. Tokman, M. Erukhimova, A. Belyanin, and J. Kono, Phys. Rev. Lett. 113, 047601 (2014).

[51] I. A. Dmitriev, A. D. Mirlin, and D. G. Polyakov, Phys. Rev. Lett. 91, 226802 (2003).

[52] I. A. Dmitriev, M. G. Vavilov, I. L. Aleiner, A. D. Mirlin, and D. G. Polyakov, Phys. Rev. B 71, 115316 (2005).

[53] V. I. Ryzhii, Sov. Phys. - Solid State 11, 2078 (1970).

[54] V. I. Ryzhii, R. A. Suris, and B. S. Shchamkhalova, Sov. Phys. - Semicond. 20, 1299 (1986).

[55] A. C. Durst, S. Sachdev, N. Read, and S. M. Girvin, Phys. Rev. Lett. 91, 086803 (2003).

[56] M. G. Vavilov and I. L. Aleiner, Phys. Rev. B 69, 035303 (2004).

[57] M. Khodas and M. G. Vavilov, Phys. Rev. B 78, 245319 (2008).

[58] T. Ando, A. B. Fowler, and F. Stern, Rev. Mod. Phys. 54, 437 (1982).

[59] I. A. Dmitriev, M. Khodas, A. D. Mirlin, D. G. Polyakov, and M. G. Vavilov, Phys. Rev. B 80, 165327 (2009).

[60] Note that in the case of linearly polarized ac field, the displacement mechanism yields an additional contribution to photoconductivity sensitive to the relative orientation of the ac and de fields. While polarization sensitivity of this kind has been studied in $[61,31]$, unambiguous observation of this displacement contribution remains a challenging task.

[61] R. G. Mani, A. N. Ramanayaka, and W. Wegscheider, Phys. Rev. B 84, 085308 (2011).

[62] The same polarization dependence is expected for nonequilibrium magneto-oscillations caused by classical memory effects $[26,63]$.

[63] I. A. Dmitriev, A. D. Mirlin, and D. G. Polyakov, Phys. Rev. B 70, 165305 (2004)

[64] Equation (3) is written for the constructive Fabry-Perot interference inside the dielectric substrate, in which case the broadening of the $\mathrm{CR}$ is maximized. In other conditions the ratio $A_{\epsilon}^{\mathrm{CRA}} / A_{\epsilon}^{\mathrm{CRI}}$ can only increase (see Supplemental Material [35]). 